\title{
REGISTROS ADICIONALES DE AVES PARA HIDALGO, MÉXICO
}

\author{
Valencia-Herverth J.*, R. VAlencia-Herverth y F. Mendoza-QuiJano \\ Instituto Tecnológico de Huejutla, Hidalgo. Km 5.5 Carret. Huejutla-Chalahuiyapa, \\ Apartado postal 94, C. P. 43000. Huejutla de Reyes, Hidalgo, MÉXICO \\ *valencia_herverth@yahoo.com.mx.
}

\begin{abstract}
RESUMEN
Presentamos 11 nuevos registros de aves procedentes de localidades del extremo noreste del estado de Hidalgo. Además, se indica la ampliación del área de distribución de Tigrisoma mexicanum. Los sitios de muestreo corresponden a parches de selva mediana subperennifolia, bosque mesófilo de montaña, vegetación riparia y áreas perturbadas. De las especies registradas, Vireo atricapilla es considerada en Peligro de Extinción por la NOM-059-ECOL-2001.
\end{abstract}

Palabras clave: Aves, nuevos registros, Vireo atricapilla, Hidalgo, México.

\begin{abstract}
We report here 11 new records of birds from several localities on the northeast of the state of Hidalgo. In addition, the extension of the area of distribution of Tigrisoma mexicanum is indicated. Sampling sites have patches of evergreen seasonal forest, cloud forest, riparian and disturbed habitats. Of the recorded species, Vireo atricapilla is considered as Endangered by the NOM-059-ECOL-2001.

Key Words: Birds, new records, Vireo atricapilla, Hidalgo, Mexico.
\end{abstract}

\section{INTRODUCCIÓN}

El conocimiento de la avifauna en nuestro país y su distribución es el resultado de muchos años de exploraciones a áreas específicas; a pesar del gran esfuerzo realizado, aún se desconoce la composición completa de distintas regiones de México (Navarro \& Benítez 1993). Sin embargo, la evidencia publicada de nuevos registros en varias zonas (Ramírez et al. 1994, Navarro \& Peterson 1999, GonzálezGarcía et al. 2004), nos muestra la parcialidad de los inventarios avifaunísticos en varios estados.

La región noreste de Hidalgo (Huasteca), ha sido poco estudiada debido, en parte, a la inaccesibilidad de la zona en un pasado reciente. Así, Bjelland \& Ray (1977) registraron un total de 125 especies en los municipios de Orizatlán, Huejutla, Tlanchinol, Calnali, Zacualtipán y Metztitlán; Howell \& Webb (1992) y Navarro et 
al. (1992) trabajaron en áreas adyacentes a Tlanchinol, registrando 41 y 116 especies respectivamente. En este trabajo, se documentan 11 nuevos registros al catálogo avifaunístico del estado de Hidalgo.

\section{MATERIAL Y MÉTODOS}

\section{Área de estudio}

El trabajo de campo se llevó a cabo en localidades de cuatro municipios del noreste del estado de Hidalgo: Huautla, Huejutla, Orizatlán y Tlanchinol (Fig. 1). El tipo de vegetación predominante en las localidades muestreadas es la selva mediana subperennifolia con diferentes grados de perturbación (Challenger 1998), con excepción de Apantlazol, Tlanchinol, donde existen relictos de bosque mesófilo de montaña.

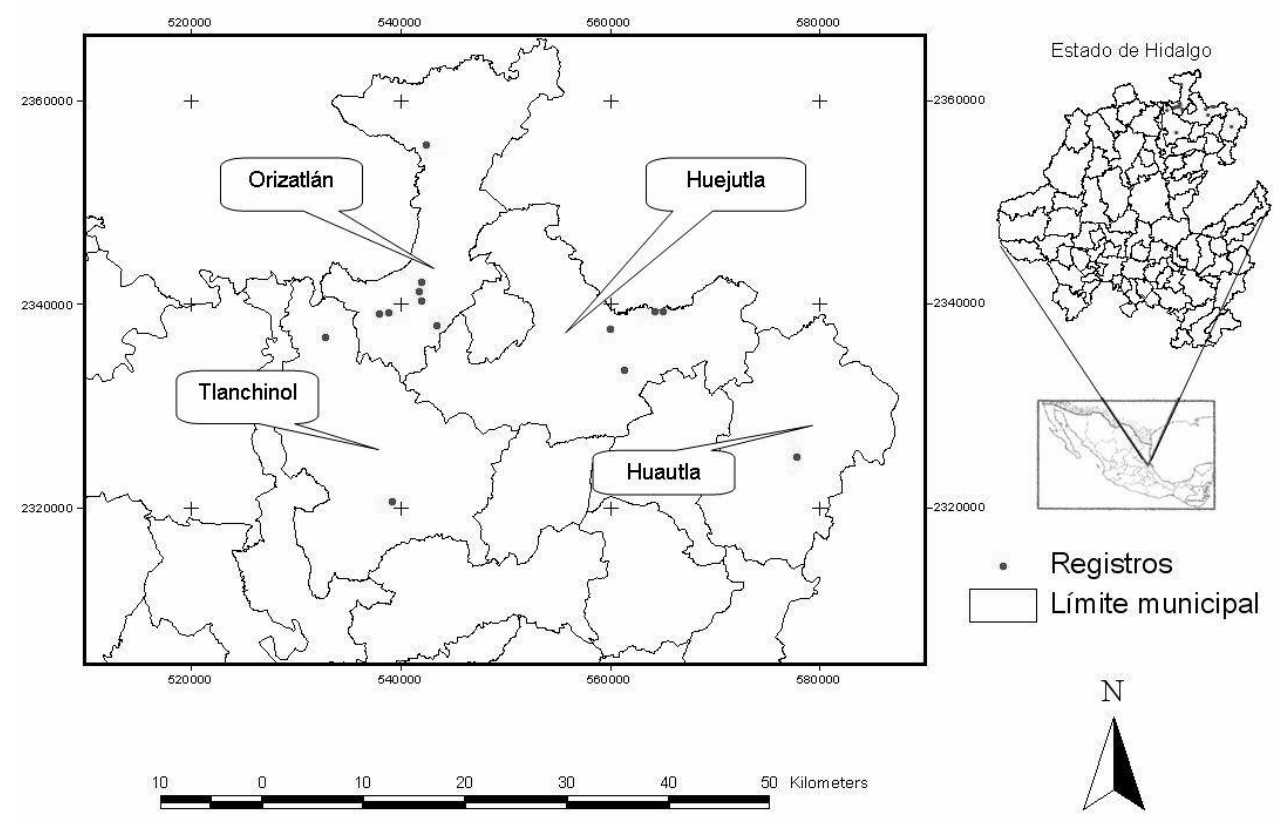

Figura 1. Localización geográfica del área de estudio, indicando las localidades mencionadas en el Cuadro 1. 


\section{Trabajo de campo}

Los muestreos se realizaron de abril de 1998 a julio de 2006, principalmente con observaciones de campo y detecciones auditivas, complementando los registros con la colecta de ejemplares. La nomenclatura y el orden taxonómico están basados en lo propuesto por la AOU $(1998,2002)$. Los nombres comunes se presentan conforme a Escalante et al. (1996). Para determinar el estatus de conservación de las especies se tomó como referencia lo establecido en la NOM-059-ECOL-2001 (SEMARNAT 2002). La estacionalidad y distribución geográfica se determinó en base a literatura (Friedman et al. 1950, Miller et al. 1957, Howell \& Webb 1995, AOU 1998). Además, se consultó la Red Mundial de Información sobre Biodiversidad (CONABIO 2006), para verificar la existencia de ejemplares colectados en el estado de Hidalgo de las especies referidas.

\section{RESULTADOS}

Se obtuvieron 11 nuevos registros para el estado de Hidalgo y una extensión del área de distribución estatal, de los cuales cinco corresponden a especies residentes reproductoras, tres visitantes de invierno, dos migratorias transitorias y dos accidentales. Entre ellas, cinco especies están consideradas bajo alguna categoría de riesgo en la NOM-059-ECOL-2001 (SEMARNAT 2002), de las cuales Vireo atricapilla está incluida en la categoría de en peligro de extinción y Crax rubra como amenazada (Anexo 1). En la Colección Ornitológica del Instituto Tecnológico de Huejutla, Hidalgo (H-AV) están depositados cuatro ejemplares y cinco fotografías (F), pertenecientes a ocho especies, las cuales se mencionan en la presente lista anotada.

Crax rubra (hocofaisán). (F) H-AV-236. El 15 de mayo de 2006 se observó un macho de aproximadamente dos años de edad, capturado por un habitante local en un fragmento de selva mediana subperennifolia adyacente a la comunidad de Temango, Tlanchinol.

Tigrisoma mexicanum (garza-tigre mexicana). El 28 de enero de 2006, fue observado un individuo juvenil en una laguna cerca del río en Santa Ana, Orizatlán. El 1 de mayo de 2006, se observó a un adulto volando y posteriormente perchando en un fragmento de selva mediana subperennifolia, en el río de La Presa, Orizatlán.

Eudocimus albus (ibis blanco). El 15 de marzo de 2003, fueron observados 15 ejemplares perchando con tres individuos de Plegadis chihi en un árbol junto al río Coacuilco en Mazaquilico, Orizatlán. El 7 de octubre de 2005, se observaron nueve 
ejemplares (cuatro juveniles y cinco adultos) forrajeando junto a Himantopus mexicanus en el río Tecoluco, Huejutla (F) H-AV-235. El 8 de noviembre de 2005, fueron observados cuatro individuos (dos juveniles y dos adultos), en el río San Pedro, San Felipe, Orizatlán. El 18 de diciembre de 2005, se observaron 11 ejemplares, en el río Coacuilco, San Felipe, Orizatlán.

Platalea ajaja (espátula rosada). Del 8 hasta el 13 de enero de 2001, se observaron seis ejemplares adultos forrajeando en el río Tecoluco, Huejutla. El 27 de enero de 2006, se observaron dos ejemplares de segundo año de edad, forrajeando en el río Tecoluco, Huejutla (F) H-AV-274.

Mycteria americana (cigüeña americana). El 30 y 31 de mayo de 2002 se observó un ejemplar buscando alimento junto a Bubulcus ibis en una lagunilla lodosa rodeada de potreros con árboles dispersos en Chalahuiyapa, Huejutla.

Parabuteo unicinctus (aguililla rojinegra). (F) H-AV-234. El 19 y 21 de noviembre de 2001 se observó a un individuo adulto perchando y acicalándose, en un área con vegetación perturbada en San Felipe, Orizatlán.

Pluvialis dominica (chorlo dominico). El 24 de marzo de 2004, se colectó (H-AV233) un adulto con plumaje no reproductivo, en el río Coacuilco cuya vegetación circundante eran potreros, fragmentos de selva mediana subperennifolia y vegetación riparia, en San Felipe, Orizatlán.

Amazona autumnalis (loro cachete amarillo). El 15 de abril de 2000, se colectó un ejemplar adulto (H-AV-038) en Chalahuiyapa, Huejutla. El 18 de enero de 2002, un ejemplar adulto fue colectado (H-AV-079) en Tzapoyo I, Orizatlán. El 21 de junio de 2005, se registraron 11 ejemplares, en un ecotono de bosque de Quercus y selva mediana subperennifolia, en la comunidad de Tres de Marzo, Orizatlán. El 14 de julio de 2006, fueron observados tres individuos perchando en una selva mediana subperennifolia, en Tamoyón I, Huautla.

Nyctibius jamaicensis (bienparado norteño). (F) H-AV-237. El 19 de mayo de 2006, fue encontrado un adulto el cual presentaba el ala izquierda lastimada en la ribera de un arroyo, con remanentes de selva mediana subperennifolia, en Xuchitl, municipio de Huejutla.

Vireo atricapilla (vireo gorra negra). (H-AV-002). El 2 de abril de 1998, fue colectado imprevistamente en una red de niebla un macho adulto (el cual murió antes de ser liberado y que por varios años estuvo mal determinado) en un bosque mesófilo de montaña, en Apantlazol, Tlanchinol. 
Campylorhynchus zonatus (matraca tropical). En San Felipe, Orizatlán el 19 de enero de 2005, se observaron dos individuos; el 2 de junio de 2005, a cuatro individuos y el 18 de marzo de 2006, se observaron dos machos. El 17 de abril de 2006, en Tamoyón I, Huautla, se observaron dos individuos. El 11 de mayo de 2006, fueron observados dos adultos y un juvenil (que era alimentado cerca del nido), en Chalahuiyapa, Huejutla.

Dendroica dominica (chipe garganta-amarilla). El 22 de enero y el 19 de febrero de 2006, en San Felipe, Orizatlán se observó un individuo, al cual se logró ver con claridad la marca en la mejilla en forma de triángulo negro, con una media luna blanca por debajo del ojo, que es muy característica de esta especie (National Geographic 2002).

\section{DISCUSIÓN}

Hasta el 2006 se tenían inventariadas 445 especies de aves para Hidalgo (MartínezMorales et al. 2007), con los 11 nuevos registros aquí presentados la lista incrementa a 456 especies. El registro del hocofaisán (C. rubra) en Hidalgo, llena un hueco en su área de distribución y es probable que esta población esté interconectada con las del estado de San Luís Potosí, o con la población registrada en la Reserva de la Biosfera Sierra Gorda de Querétaro, por Eitniear et al. (2000); siendo esta especie un indicador de la calidad del hábitat ya que se encontró en un área con selva madura. En contraste, tres especies, el ibis blanco (E. albus), la espátula rosada (P. ajaja) y el chipe gargantaamarilla $(D$. dominica) se localizaron en áreas perturbadas, estás no están delineadas en distribución para la zona en los mapas de Howell \& Webb (1995). Son escasos los registros existentes de la cigüeña americana (M. americana) tierra adentro (Friedmann et al. 1950), por lo que se considera un registro accidental al igual que el de la aguililla rojinegra ( $P$. unicinctus) la cual se registró en un hábitat inusual para la especie. El registro del chorlo dominico $(P$. dominica) es uno de los pocos que se tienen para México, ya que es un ave difícil de observar en migración (Peterson \& Chalif 1989), los registros han sido principalmente en las costas del Pacífico (Navarro \& Peterson 1999) y en la vertiente del Golfo solamente se tiene registrado para los estados de Puebla y Veracruz (Chávez et al. 1996, Navarro et al. 2004, Montejo \& McAndrews 2006). El loro cachete amarillo (A. autumnalis) y el bienparado norteño ( $N$. jamaicensis) anteriormente fueron registrados en los estados vecinos de San Luis Potosí y Veracruz, así, era de esperarse su presencia en Hidalgo. El vireo gorra negra ( $V$. atricapilla) es probable que el individuo era transitorio o se hallaba fuera del rango migratorio, aunque ocasionalmente durante la migración se encuentra en Tamaulipas (Navarro 2000); ésta es una especie migratoria de invierno, está considerada en 
peligro de extinción en la NOM-059-ECOL-2001, la U. S. Fish and Wildlife Service y en categoría "A" por la IUCN (Collar et al. 1992), ya que sus poblaciones han declinado drásticamente, por tanto se sugiere realizar más monitoreos en los bosques mesófilos de montaña adyacentes a Tlanchinol para conocer sí la especie utiliza este hábitat en su ruta migratoria, o como se mencionó anteriormente fue un ejemplar fuera del rango migratorio. Se reafirma la hipótesis planteada por Miller et al. (1957), acerca de la probable presencia de la matraca tropical (C. zonatus) en el sureste de San Luis Potosí, ya que la localidad de San Felipe, Orizatlán se encuentra a $15 \mathrm{~km}$ del límite estatal. En el caso de la garza-tigre mexicana (T. mexicanum) se tiene un registro histórico de 1834 mencionado por Friedmann et al. (1950) para el sur del estado en Real del Monte, por lo que estos registros significan los primeros para Hidalgo en más de 170 años y se constituye como una extensión del área en el estado hacia el NE.

\section{AGRADECIMIENTOS}

Agradecemos de manera muy especial a la gente de las comunidades que fueron visitadas durante el trabajo de campo. Al Dr. Miguel A. Martínez-Morales, a la Dra. Patricia Escalante y a los tres revisores anónimos, por sus valiosos comentarios y sugerencias realizadas al manuscrito. Los autores agradecen el registro de colecciones científicas museográficas públicas o privadas de especímenes silvestres expedido por SEMARNAT (HGO-AV-1260420) al ITH.

\section{LITERATURA CITADA}

AOU (American Ornithologist's Union). 1998. Check-list of North American Birds. $7^{\text {th }}$ ed American Ornithologist's Union, Washington, D.C.

http://www.aou.org/aou/birdlist.htm.

Bjelland, A.D. \& J.C. Ray. 1977. Birds collected in the state of Hidalgo, Mexico. Occ. Pap. Mus. Tex. Tech. Univ. 46:1-32.

Challenger, A. 1998. Utilización y conservación de los ecosistemas terrestres de México. Pasado, presente y futuro. CONABIO, IBUNAM y Sierra Madre, México, D. F.

Chávez C., N., M.A. Gurrola H. \& J.A. García L. 1996. Catálogo de aves no passeriformes de la colección ornitológica del Instituto de Biología, UNAM. Cuadernos de Inst. Biol. (30): 1-145.

Collar, N.J., L.P. Gonzaga, N. Krabbe, A. Madroño N., L.G. Naranjo, T.A. Parker III \& C. Wege. 1992. Threatened Birds of the Americas, the ICBP/IUCN Red Book, Part 2. International Council for Birds Preservation Cambridge, U. K. Third edition.

CONABIO (Comisión Nacional para el Conocimiento y Uso de la Biodiversidad). 2006. Red Mundial de Información sobre Biodiversidad. <http://www.conabio.gob.mx/remib/ 
doctos/remibnodosdb.html >. Fecha de consulta: 14 de Septiembre de 2006. Fecha ultima de modificación: 28/02/2002.

Eitniear, J.C., A. Aragón, V. González, R. Pedraza \& J.T. Baccus. 2000. New Galliformes for the mexican state of Querétaro. Cotinga. 13:10-13.

Escalante P, P., A.M. Sada \& J. Robles G. 1996. Listado de nombres comunes de las aves de México. CONABIO-Sierra Madre, México, D. F.

Friedmann, H., L. Griscom \& R.T. Moore. 1950. Distributional check-list of the birds of Mexico. Part I. Pacific Coast Avif. 29: 1- 202.

González-García, F., F. Puebla, S. Barrios, M.N. Fajardo \& H. Gómez de Silva. 2004. Información adicional sobre la avifauna de los estados de Hidalgo y Querétaro, México, incluyendo nuevos registros estatales. Cotinga 22: 56-64.

Howell, S.N.G. \& S. Webb. 1992. A little known cloud forest in Hidalgo, Mexico. Euphonia $1: 7-11$.

Howell, S.N.G. \& S. Webb. 1995. A guide to the birds of Mexico and Northern Central America. Oxford University Press, New York.

Martínez-Moralez, M.A., R. Ortiz-Pulido, B. de la Barreda, I.L. Zuria, J. Bravo-Cadena \& J. Valencia-Herverth. 2007. Hidalgo. Pp. 49-95. In: R. Ortiz-Pulido, A. NavarroSigüenza, H. Gómez de Silva, O. Rojas Soto y T.A. Peterson (Eds.). Avifaunas estatales de México. CIPAMEX, Pachuca, Hidalgo, México.

Miller, A.H., H. Friedman, L. Griscom \& R.T. Moore. 1957. Distributional check-list of the birds of Mexico. Part II. Pacific Coast Avif. 33:1-436.

Montejo D., J. \& A. McAndrews. 2006. Listado de las aves de Veracruz, México/Check-list of the Birds of Veracruz, Mexico. Boletín de Divulgación No. 1/Outreach Bulletin No. 1. Endémicos Insulares, A. C. Veracruz, Veracruz, México.

National Geographic Society. 2002. Field guide to the birds of North America. Fourth edition. National Geographic Society, Washington, D.C.

Navarro S., A.G. 2000. Vireo gorra negra (Vireo atricapillus). Pp. 301-302. In: G. Ceballos y L. Márquez V. (Eds.). Las aves de México en peligro de extinción. CONABIO, INE, UNAM y FCE, México.

Navarro S., A.G. \& A.T. Peterson. 1999. Extensión del área de distribución de aves en el oeste de Guerrero, México. An. Inst. Biol. 70:41-50.

Navarro S., A.G. \& H. Benítez. 1993. Patrones de riqueza y endemismo de las aves. Revista Ciencias, No. Especial 7:45-54.

Navarro S., A.G., H. Benítez \& B.E. Hernández. 1992. Avifauna de los bosques de montaña del noreste del estado de Hidalgo. Reporte de Biología de Campo, Facultad de Ciencias UNAM.

Navarro S., A.G., H. Garza-Torres, S. López de Aquino, O. Rojas-Soto \& L. SánchezGonzález. 2004. Patrones biogeográficos de la avifauna. Pp. 439-467. In: I. Luna, J. J. Morrone y D. Espinosa (Eds.). Biodiversidad de la Sierra Madre Oriental, Las prensas de Ciencias, México, D. F.

Peterson, R.T. \& E.L. Chalif. 1989. Aves de México, Guía de Campo. Edit. Diana, México, D. F. 
Valencia, Valencia \& Mendoza: Registros de aves para Hidalgo, México

Ramírez B, P., A. DeSucre-Medrano, A.G. Navarro, P.J. Romo \& H. Castro. 1994. Winter specimen of the Common Loon (Gavia immer) from the state of Hidalgo, México. Southwest. Nat. 39:394-395.

SEMARNAT (Secretaría de Medio Ambiente y Recursos Naturales). 2002. Norma Oficial Mexicana NOM-059-ECOL-2001, Protección ambiental -especies nativas de México y de flora y fauna silvestres- categorías de riesgo y especificaciones para su inclusión, exclusión o cambio- lista de especies en riesgo. Diario Oficial de la Federación.

Recibido: 5 de mayo de 2007

Aceptado: 11 de enero de 2008 
Anexo 1. Localización geográfica y estatus de conservación de los nuevos registros de aves para el estado de Hidalgo.

\begin{tabular}{|c|c|c|c|c|c|c|c|c|}
\hline Especie & Localidad & Municipio & Latitud N & $\begin{array}{c}\text { Longitud } \\
0\end{array}$ & $\begin{array}{l}\text { Altitud } \\
(\mathrm{msnm})\end{array}$ & Fecha & $\begin{array}{l}\text { Tipode } \\
\text { registro }\end{array}$ & $\begin{array}{c}\text { Estatus de } \\
\text { conserva ción }\end{array}$ \\
\hline Crax rubra & Temango & Tlanchinol & $21^{\circ} 07^{\prime} 58^{\prime \prime}$ & $98^{\circ} 41^{\prime} 20^{\prime \prime}$ & 498 & $15 / 05 / 2006$ & $\mathrm{~F}$ & $\mathrm{~A}$ \\
\hline \multirow{2}{*}{$\begin{array}{l}\text { Tigrisoma } \\
\text { mexicanum }\end{array}$} & Santa Ana & Orizatlán & $21^{\circ} 10^{\prime} 53^{\prime \prime}$ & $98^{\circ} 35^{\prime} 51^{\prime \prime}$ & 200 & $28 / 01 / 2006$ & $\mathrm{O}$ & $\operatorname{Pr}$ \\
\hline & La Presa & Orizatlán & $21^{\circ} 09^{\prime} 13^{\prime \prime}$ & $98^{\circ} 38^{\prime} 18^{\prime \prime}$ & 200 & $1 / 05 / 2006$ & $\mathrm{O}$ & \\
\hline \multirow[t]{4}{*}{ Eudocimus albus } & $\begin{array}{l}\text { Mazaqu ilico (Río } \\
\text { Coacuilco) }\end{array}$ & Orizatlán & $21^{\circ} 08^{\prime} 33^{\prime \prime}$ & $98^{\circ} 35^{\prime} 00^{\prime \prime}$ & 140 & $15 / 03 / 2003$ & $\mathrm{O}$ & \\
\hline & $\begin{array}{l}\text { Huejutla de Reyes } \\
\text { (Río Te coluco) }\end{array}$ & Huejutla & $21^{\circ} 08^{\prime} 33^{\prime \prime}$ & $98^{\circ} 25^{\prime} 05^{\prime \prime}$ & 158 & $7 / 10 / 2005$ & $\mathrm{~F}$ & \\
\hline & $\begin{array}{l}\text { San Felipe (Río } \\
\text { San Pedro) }\end{array}$ & Orizatlán & $21^{\circ} 10^{\prime} 27^{\prime \prime}$ & $98^{\circ} 36^{\prime} 00^{\prime \prime}$ & 150 & $8 / 11 / 2005$ & $\mathrm{O}$ & \\
\hline & $\begin{array}{l}\text { San Felipe (Río } \\
\text { Coacuilco) }\end{array}$ & Orizatlán & $21^{\circ} 09^{\prime} 53^{\prime \prime}$ & $98^{\circ} 35^{\prime} 51^{\prime \prime}$ & 170 & $18 / 12 / 2005$ & $\mathrm{O}$ & \\
\hline \multirow[t]{7}{*}{ Platalea ajaja } & $\begin{array}{l}\text { Huejutla de Reyes } \\
\text { (Río Te coluco) }\end{array}$ & Huejutla & $21^{\circ} 08^{\prime} 22^{\prime \prime}$ & $98^{\circ} 25^{\prime} 11^{\prime \prime}$ & 158 & $8 / 01 / 2001$ & $\mathrm{O}$ & \\
\hline & $\begin{array}{l}\text { Huejutla de Reyes } \\
\text { (Río Te coluco) }\end{array}$ & Huejutla & $21^{\circ} 08^{\prime} 22^{\prime \prime}$ & $98^{\circ} 25^{\prime} 11^{\prime \prime}$ & 158 & $9 / 01 / 2001$ & $\mathrm{O}$ & \\
\hline & $\begin{array}{l}\text { Huejutla de Reyes } \\
\text { (Río Te coluco) }\end{array}$ & Huejutla & $21^{\circ} 08^{\prime} 22^{\prime \prime}$ & $98^{\circ} 25^{\prime} 11^{\prime \prime}$ & 158 & $10 / 01 / 2001$ & $\mathrm{O}$ & \\
\hline & $\begin{array}{l}\text { Huejutla de Reyes } \\
\text { (Río Te coluco) }\end{array}$ & Huejutla & $21^{\circ} 08^{\prime} 22^{\prime \prime}$ & $98^{\circ} 25^{\prime} 11^{\prime \prime}$ & 158 & $11 / 01 / 2001$ & $\mathrm{O}$ & \\
\hline & $\begin{array}{l}\text { Huejutla de Reyes } \\
\text { (Río Te coluco) }\end{array}$ & Huejutla & $21^{\circ} 08^{\prime} 22^{\prime \prime}$ & $98^{\circ} 25^{\prime} 11^{\prime \prime}$ & 158 & $12 / 01 / 2001$ & $\mathrm{O}$ & \\
\hline & $\begin{array}{l}\text { Huejutla de Reyes } \\
\text { (Río Te coluco) }\end{array}$ & Huejutla & $21^{\circ} 08^{\prime} 22^{\prime \prime}$ & $98^{\circ} 25^{\prime} 11^{\prime \prime}$ & 158 & $13 / 01 / 2001$ & $\mathrm{O}$ & \\
\hline & $\begin{array}{l}\text { Hu ejutla de Reyes } \\
\text { (Río Te coluco) }\end{array}$ & Huejutla & $21^{\circ} 08^{\prime} 22^{\prime \prime}$ & $98^{\circ} 25^{\prime} 11^{\prime \prime}$ & 158 & $27 / 01 / 2007$ & $\mathrm{~F}$ & \\
\hline \multirow[t]{2}{*}{ Mycteria americana } & Chalahuiyapa & Huejutla & $21^{\circ} 09^{\prime} 16^{\prime \prime}$ & $98^{\circ} 22^{\prime} 36^{\prime \prime}$ & 155 & $30 / 05 / 2002$ & $\mathrm{O}$ & $\operatorname{Pr}$ \\
\hline & Chalahuiyapa & Huejutla & $21^{\circ} 09^{\prime} 16^{\prime \prime}$ & $98^{\circ} 22^{\prime} 36^{\prime \prime}$ & 155 & $31 / 05 / 2002$ & $\mathrm{O}$ & \\
\hline \multirow[t]{2}{*}{ Parabuteo unic inctus } & San Felipe & Orizatlán & $21^{\circ} 09^{\prime} 16^{\prime \prime}$ & $98^{\circ} 37^{\prime} 43^{\prime \prime}$ & 150 & $19 / 11 / 2001$ & $\mathrm{O}$ & $\operatorname{Pr}$ \\
\hline & San Felipe & Orizatlán & $21^{\circ} 09^{\prime} 16^{\prime \prime}$ & $98^{\circ} 37^{\prime} 43^{\prime \prime}$ & 150 & $21 / 11 / 2001$ & $\mathrm{~F}$ & \\
\hline Pluvialis dominica & $\begin{array}{l}\text { San Felipe (Río } \\
\text { Coacuilco) }\end{array}$ & Orizatlán & $21^{\circ} 09^{\prime} 53^{\prime \prime}$ & $98^{\circ} 35^{\prime} 51^{\prime \prime}$ & 170 & $24 / 03 / 2004$ & $\mathrm{C}$ & \\
\hline \multirow[t]{4}{*}{ Amazona autumnalis } & Chalahuiyapa & Huejutla & $21^{\circ} 09^{\prime} 16^{\prime \prime}$ & $98^{\circ} 22^{\prime} 11^{\prime \prime}$ & 150 & $15 / 04 / 2000$ & $\mathrm{C}$ & \\
\hline & Tzapo llo I & Orizatlán & $21^{\circ} 18^{\prime} 11^{\prime \prime}$ & $98^{\circ} 35^{\prime} 34^{\prime \prime}$ & 160 & $18 / 01 / 2002$ & $\mathrm{C}$ & \\
\hline & Tres de Marzo & Orizatlán & $21^{\circ} 09^{\prime} 16^{\prime \prime}$ & $98^{\circ} 37^{\prime} 44^{\prime \prime}$ & 180 & $21 / 06 / 2005$ & $\mathrm{O}$ & \\
\hline & Tamoyón I & Huautla & $21^{\circ} 01^{\prime} 30^{\prime \prime}$ & $98^{\circ} 14^{\prime} 36^{\prime \prime}$ & 200 & $14 / 07 / 2006$ & $\mathrm{O}$ & \\
\hline Nyctibius jamaicensis & Xuchitl & Huejutla & $21^{\circ} 06^{\prime} 11^{\prime \prime}$ & $98^{\circ} 24^{\prime} 22^{\prime \prime}$ & 160 & $19 / 05 / 2006$ & $\mathrm{~F}$ & \\
\hline Vireo atricapilla & Apantlazol & Tlanchinol & $20^{\circ} 59^{\prime} 14^{\prime \prime}$ & $98^{\circ} 37^{\prime} 36^{\prime \prime}$ & 1550 & $2 / 04 / 1998$ & $\mathrm{C}$ & $\mathrm{P}$ \\
\hline \multirow{5}{*}{$\begin{array}{l}\text { Campylorhynchus } \\
\text { zonatus }\end{array}$} & San Felipe & Orizatlán & $21^{\circ} 09^{\prime} 16^{\prime \prime}$ & $98^{\circ} 37^{\prime} 43^{\prime \prime}$ & 150 & $19 / 01 / 2005$ & $\mathrm{O}$ & \\
\hline & San Felipe & Orizatlán & $21^{\circ} 09^{\prime} 16^{\prime \prime}$ & $98^{\circ} 37^{\prime} 43^{\prime \prime}$ & 150 & $2 / 06 / 2005$ & $\mathrm{O}$ & \\
\hline & San Felipe & Orizatlán & $21^{\circ} 09^{\prime} 16^{\prime \prime}$ & $98^{\circ} 37^{\prime} 43^{\prime \prime}$ & 150 & $18 / 03 / 2006$ & $\mathrm{O}$ & \\
\hline & Tamoyón I & Huautla & $21^{\circ} 01^{\prime} 30^{\prime \prime}$ & $98^{\circ} 14^{\prime} 36^{\prime \prime}$ & 200 & $17 / 04 / 2006$ & $\mathrm{O}$ & \\
\hline & Chalahuiyapa & Huejutla & $21^{\circ} 09^{\prime} 16^{\prime \prime}$ & $98^{\circ} 22^{\prime} 36^{\prime \prime}$ & 155 & $11 / 05 / 2006$ & $\mathrm{O}$ & \\
\hline \multirow[t]{2}{*}{ Dendroica dominica } & San Felipe & Orizatlán & $21^{\circ} 09^{\prime} 16^{\prime \prime}$ & $98^{\circ} 37^{\prime} 43^{\prime \prime}$ & 150 & $22 / 01 / 2006$ & $\mathrm{O}$ & \\
\hline & San Felipe & Orizatlán & $21^{\circ} 09^{\prime} 16^{\prime \prime}$ & $98^{\circ} 37^{\prime} 43^{\prime \prime}$ & 150 & $19 / 02 / 2006$ & $\mathrm{O}$ & \\
\hline
\end{tabular}

$\mathrm{O}=$ Observación, $\mathrm{F}=$ Fotografía, $\mathrm{C}=$ Colecta; $\mathrm{P}=$ En peligro de extinción, $\mathrm{A}=$ Amenazada, $\mathrm{Pr}=$ Protección especial. 
